CRYSTALLOGRAPHIC COMMUNICATIONS

ISSN 2056-9890

Reçu le 25 mars 2016

Accepté le 19 avril 2016

Edité par A. Van der Lee, Université de Montpellier II, France

Keywords: crystal structure; sodium-iron molybdate; occupational substitution.

CCDC reference: 1423015

Supporting information: this article has supporting information at journals.iucr.org/e
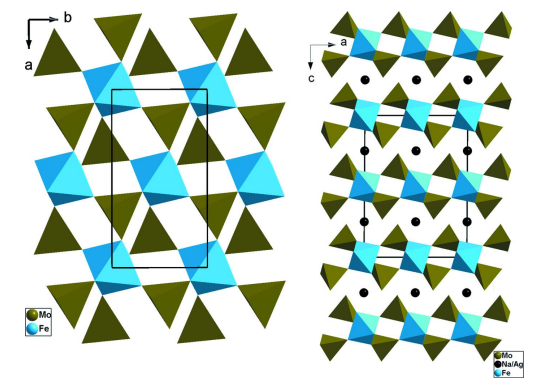

OPEN $\odot$ ACCESS

\section{Synthèse, étude et validation structurale d'un triple bis-molybdate en couches, $\mathrm{Ag}_{0.60} \mathrm{Na}_{0.40} \mathrm{Fe}\left(\mathrm{MoO}_{4}\right)_{2}$ lié à yavapaiite}

\author{
Amira Souilem* et Mohamed Faouzi Zid
}

Laboratoire de Matériaux et Cristallochimie, Faculté des Sciences de Tunis, Université de Tunis El Manar, 2092 El Manar Tunis, Tunisia. *Correspondence e-mail: souilem_amira@yahoo.fr

The title compound, $\mathrm{Ag}_{0.60} \mathrm{Na}_{0.40} \mathrm{Fe}\left(\mathrm{MoO}_{4}\right)_{2}$ \{ silver sodium iron(III) bis[molybdate(VI)]\}, was synthesized by a solid-state reaction. The structure is built up from $\mathrm{FeO}_{6}$ octahedra and $\mathrm{MoO}_{4}$ tetrahedra sharing corners to form a twodimensional framework parallel to the (001) plane. The occupationally disordered $(\mathrm{Ag} / \mathrm{Na})^{+}$cations are located in the interlayer space. These $\left[\mathrm{Fe}\left(\mathrm{MoO}_{4}\right)_{2}{ }^{-}\right]_{n}$ layers are similar to those in the isotypic yavapaiite-related structure of $\mathrm{NaFe}\left(\mathrm{MoO}_{4}\right)_{2}$. All atoms are on general positions except for Fe (site symmetry $\overline{1}$ ) and $\mathrm{Ag} / \mathrm{Na}$ (site symmetry 2 ). The $\mathrm{Ag} / \mathrm{Na}$ occupancies of the latter site were refined to 0.597 (4):0.403 (5). The title structure is compared to similar structures.

\section{Contexte cristallo-chimique}

La recherche de nouveaux matériaux, pouvant être utilisés comme source d'énergie cathodique, a encouragé de nombreuses équipes de recherche de synthétiser des composés à charpente ouverte. En effet, la jonction octaèdres-tétraèdres dans ce type de matériaux s'avère un chemin efficace pour l'élaboration de certains conducteurs ioniques: $\mathrm{Li}_{3} \mathrm{Fe}_{3}\left(X \mathrm{O}_{4}\right)_{3}$ $\left(X=\mathrm{P}\right.$, As; Masquelier et al., 1998), $\mathrm{Na}_{7} \mathrm{Fe}_{4}\left(\mathrm{AsO}_{4}\right)_{6}$ (Masquelier et al., 1995), $\mathrm{LiFePO}_{4}$ (Padhi et al., 1997), $\mathrm{KFeAs}_{2} \mathrm{O}_{7}$ (Ouerfelli et al., 2007), $\mathrm{Ag}_{2} \mathrm{VP}_{2} \mathrm{O}_{8}$ (Daidouh et al., 1997), $\mathrm{Na}_{2} \mathrm{CoP}_{2} \mathrm{O}_{7}$ (Sanz et al., 1999), $\alpha-\mathrm{Na}_{3} \mathrm{Al}_{2}\left(\mathrm{AsO}_{4}\right)_{3}$ (D'Yvoire et al., 1986) et notamment la famille Nasicon $\mathrm{Na}_{3} \mathrm{Zr}_{2} \mathrm{PSi}_{2} \mathrm{O}_{12}$ (Goodenough et al., 1976; D'Yvoire et al., 1983; Boilot et al., 1987).

L'élargissement des dimensions des canaux ainsi que l'occupation partielle des sites dans la structure devraient être deux autres facteurs favorables à la mobilité des cations monovalents. Nous avons alors choisi le molybdène (+VI) de rayon ionique supérieur à celui du silicium (+IV), du phosphore $(+V)$ ou de l'arsenic $(+V)$ d'après Shannon (1976) pour qu'il occupe les sites tétraédriques dans la structure. Plusieurs nouvelles phases ont été élaborées dans lesquelles les cavités octaédriques sont occupées seulement par des ions bi- ou trivalents ( $\mathrm{Mg}, \mathrm{Mn}, \mathrm{Fe}, \mathrm{Co}$, etc.) notamment: $\beta$ - $\mathrm{Li}_{0.37} \mathrm{Na}_{0.63^{-}}$ $\mathrm{Fe}\left(\mathrm{MoO}_{4}\right)_{2}$ de type wolframite (Souilem et al., 2014), $\left(\mathrm{Na}_{0.4} \mathrm{Li}_{0.6}\right)\left(\mathrm{Fe}, \mathrm{Li}_{2}\right)\left(\mathrm{MoO}_{4}\right)_{3}$ de type lyonsite (Souilem et al., 2015), $\quad \mathrm{KNa}_{5} \mathrm{Mn}_{3}\left(\mathrm{MoO}_{4}\right)_{6} \quad$ (Bouzidi et al., 2015) et $\mathrm{K}_{0.13} \mathrm{Na}_{3.87} \mathrm{MgMo}_{3} \mathrm{O}_{12}$ (Ennajeh et al., 2015) de type alluaudite. Dans ce cadre un nouveau matériau de formulation $\mathrm{Ag}_{0.6} \mathrm{Na}_{0.4} \mathrm{Fe}\left(\mathrm{MoO}_{4}\right)_{2}$ à charpente bidimensionnelle a été élaboré par réaction à l'état solide à $1173 \mathrm{~K}$. Il s'avère appartenant à la famille yavapaiite et est isostructural au composé 


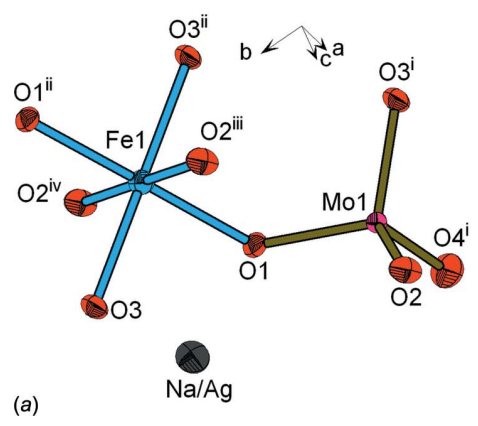

Figure 1

Représentation: $(a)$ atomique complète des polyèdres de coordination dans l'unité asymétrique de $\mathrm{Ag}_{0.60} \mathrm{Na}_{0.40} \mathrm{FeMo}_{2} \mathrm{O}_{8} ;(b)$ polyédrique de la double unité $\mathrm{Mo}_{2} \mathrm{Fe}_{2} \mathrm{O}_{16}$. Les ellipsoides dans $(a)$ ont été définis avec $50 \%$ de probabilité. [Codes de symétrie: (i) $x, y-1, z$; (ii) $-x+1,-y+1$, $-z+1$; (iii) $-x+\frac{3}{2},-y+\frac{1}{2},-z+1$; (iv) $x-\frac{1}{2}, y+\frac{1}{2}, z$.]

$\mathrm{NaFe}\left(\mathrm{MoO}_{4}\right)_{2}(\mathrm{Klevtsova}, 1975)$ ayant comme paramètres de maille, $a=9.87, b=5.31, c=13.57 \AA, \beta=90.4^{\circ}$ et groupe d'espace $C 2 / c$.

\section{Commentaire structural}

L'unité asymétrique dans $\mathrm{Ag}_{0.6} \mathrm{Na}_{0.4} \mathrm{Fe}\left(\mathrm{MoO}_{4}\right)_{2}$ est formée d'un tétraèdre $\mathrm{MoO}_{4}$ relié au moyen d'un sommet à un octaèdre $\mathrm{FeO}_{6}$ et de deux cations monovalents partageant le même site cristallographique. Les polyèdres de coordination, en ajoutant les atomes d'oxygène équivalents, ainsi que les ions monovalents, sont représentés sous forme d'une unité à Fig. 1a. Deux unités se regroupent par ponts mixtes de type Mo-O-Fe pour former une unité formulaire double cyclique $\mathrm{Mo}_{2} \mathrm{Fe}_{2} \mathrm{O}_{16}$ dans laquelle la compensation de charges est assurée par les cations $\mathrm{Ag}^{+} / \mathrm{Na}^{+}$(Fig. 1b). La structure peut être donc décrite moyennant des chaînes classiques de type $\mathrm{MoFeO}_{8}$ formées par l'association, par sommets, des unités

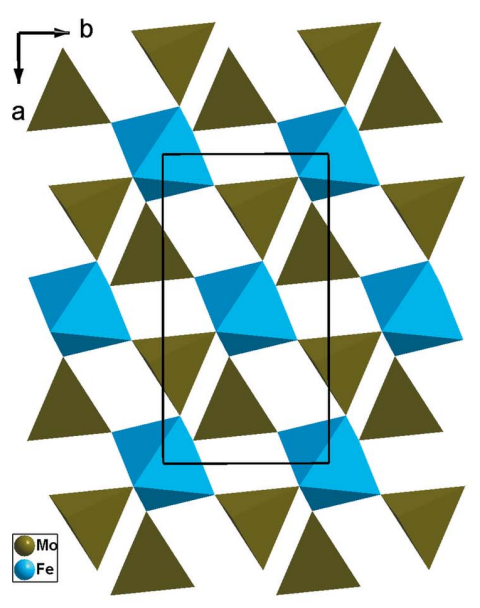

(a)

Figure 2

Projections: $(a)$ selon l'axe $c$, d'une couche infinie de type $\left(\mathrm{FeMo}_{2} \mathrm{O}_{8}\right)_{n}$ dans $\mathrm{Ag}_{0.60} \mathrm{Na}_{0.40} \mathrm{FeMo}_{2} \mathrm{O}_{8}$; (b) selon $b$, de la structure mettant en évidence l'espace intercouches où logent les cations monovalents.
Tableau 1

Longueurs de liaison sélectionnés (Å).

\begin{tabular}{llll}
\hline Mo1-O4 $^{\text {i }}$ & $1,711(4)$ & $\mathrm{Ag} 1-\mathrm{O} 4^{\mathrm{ii}}$ & $2,499(4)$ \\
Mo1-O3 $^{\mathrm{i}}$ & $1,754(3)$ & $\mathrm{Fe} 1-\mathrm{O} 2^{\mathrm{iii}}$ & $1,957(3)$ \\
$\mathrm{Mo1}-\mathrm{O} 2$ & $1,788(3)$ & $\mathrm{Fe} 1-\mathrm{O} 2^{\mathrm{iv}}$ & $1,957(3)$ \\
$\mathrm{Mo1}-\mathrm{O} 1$ & $1,797(3)$ & $\mathrm{Fe} 1-\mathrm{O} 1$ & $1,981(3)$ \\
$\mathrm{Ag} 1-\mathrm{O} 1$ & $2,367(3)$ & $\mathrm{Fe} 1-\mathrm{O} 1^{\mathrm{v}}$ & $1,981(3)$ \\
$\mathrm{Ag} 1-\mathrm{O} 1^{\mathrm{ii}}$ & $2,367(3)$ & $\mathrm{Fe} 1-\mathrm{O} 3$ & $1,994(3)$ \\
$\mathrm{Ag} 1-\mathrm{O} 4$ & $2,499(4)$ & $\mathrm{Fe} 1-\mathrm{O} 3^{\mathrm{v}}$ & $1,994(3)$ \\
\hline
\end{tabular}

Codes de symétrie: (i) $x, y-1, z$; (ii) $-x+1, y,-z+\frac{3}{2}$; (iii) $x-\frac{1}{2}, y+\frac{1}{2}, z$; (iv) $-x+\frac{3}{2},-y+\frac{1}{2},-z+1 ;(\mathrm{v})-x+1,-y+1,-z+1$.

asymétriques $\mathrm{MoFeO}_{9}$. Une disposition particulière, en trans, des tétraèdres permet la jonction des chaînes dans le plan $(a b)$ et conduit à des couches (Fig. $2 a$ ). Il en résulte une structure à charpente bidimensionnelle où les cations $\mathrm{Ag}^{+} / \mathrm{Na}^{+}$se situent dans l'espace intercouches (Fig. 2b). Les distances moyennes dans la structure étudiée pour $\mathrm{Mo}-\mathrm{O}$ et $\mathrm{Fe}-\mathrm{O}$ sont égales à 1.763 (3) et 1.977 93) Å, respectivement. Elles sont conformes à celles rencontrées dans les structures analogues $A \mathrm{Fe}\left(\mathrm{MoO}_{4}\right)_{2}(A=$ cation monovalent $)$. Les cations $\mathrm{Ag}^{+} / \mathrm{Na}^{+}$ occupant les mêmes sites cristallographiques sont entourés par quatre atomes d'oxygène respectivement. La distance moyenne $A^{+}-\mathrm{O}\left(A^{+}=\mathrm{Ag}^{+} / \mathrm{Na}^{+}\right)$est égale à $2.433(5) \AA$ (tableau 1). Elle est de même ordre de grandeur que celles $\mathrm{Na}-\mathrm{O}(2.426$ et $2.461 \AA$ ) rencontrées respectivement dans les deux composés isotypes: $\mathrm{NaFe}\left(\mathrm{MoO}_{4}\right)_{2}$ (Klevtsova, 1975) et $\mathrm{NaAl}\left(\mathrm{MoO}_{4}\right)_{2}($ Kolitsch et al., 2003) de type yavapaiite.

Les octaèdres $\mathrm{FeO}_{6}$ et les tétraèdres $\mathrm{MoO}_{4}$ sont relativement réguliers. Les indices de distorsion respectifs, calculés à partir des formules de Baur (1974) et Wildner (1992), sont de l'ordre de 0.6 et $1.7 \%$. Ces grandeurs sont proches de celles calculées dans le cas de $\mathrm{NaFe}\left(\mathrm{MoO}_{4}\right)_{2}$, respectivement 1.6 et $4.2 \%$ pour l'octaèdre $\mathrm{FeO}_{6}$ et le tétratèdre $\mathrm{MoO}_{4}$. La substitution partielle de l'ion $\mathrm{Na}^{+}$par $\mathrm{Ag}^{+}$, cation polarisable, dans la structure a eu donc un léger effet sur la distorsion des polyèdres. De plus, le calcul des valences des liaisons (BVS), en utilisant la formule empirique de Brown (Brown \& Altermatt, 1985) et les facteurs géométriques déduits de l'étude structurale, conduit aux valeurs des charges des ions suivants: Mo1 (5.98), Fe1 (3.12), Ag1/Na1 (1.03) ce qui confirme les degrés d'oxydation des différents ions attendus dans la structure.

La comparaison de la structure de $\mathrm{Ag}_{0.6} \mathrm{Na}_{0.4} \mathrm{Fe}\left(\mathrm{MoO}_{4}\right)_{2}$ avec celles rencontrées dans la littérature et construites au moyen des unités $M X \mathrm{O}_{9}(M=$ métal de transition et $X=\mathrm{P}$, As ou Mo), les groupements $M_{2} X_{2} \mathrm{O}_{16}$, les chaînes classiques $M \mathrm{XO}_{8}$, révèle une certaine filiation entre les structures de formulation analogue bien que les phases considérées présentent des symétries différentes notamment: $\mathrm{Ba}_{3} \mathrm{Mo}_{2} \mathrm{O}_{2}\left(\mathrm{PO}_{4}\right)_{4}, \mathrm{KMo}\left(\mathrm{PO}_{4}\right)_{2}, A \mathrm{Fe}\left(\mathrm{MoO}_{4}\right)_{2}$ et $A \mathrm{MoO}_{2} \mathrm{AsO}_{4}$ ( $A=\mathrm{Li}$, Na ou $\mathrm{Cs}$ ).

En effet, dans le composé $\mathrm{Ba}_{3} \mathrm{Mo}_{2} \mathrm{O}_{2}\left(\mathrm{PO}_{4}\right)_{4}$ (Ledain et al., 1996) de symétrie triclinique $(P \overline{1})$ les unités $\mathrm{MoPO}_{9}$ se lient au moyen de ponts mixtes Mo-O-P pour former des rubans et conduisent à une charpente unidimensionnelle (Fig. 3). Dans la phase $\mathrm{KMo}\left(\mathrm{PO}_{4}\right)_{2}$ (Gueho et al., 1992) [isotype à $\mathrm{KNb}\left(\mathrm{PO}_{4}\right)_{2}$; Linde et al., 1980] de symétrie monoclinique, 


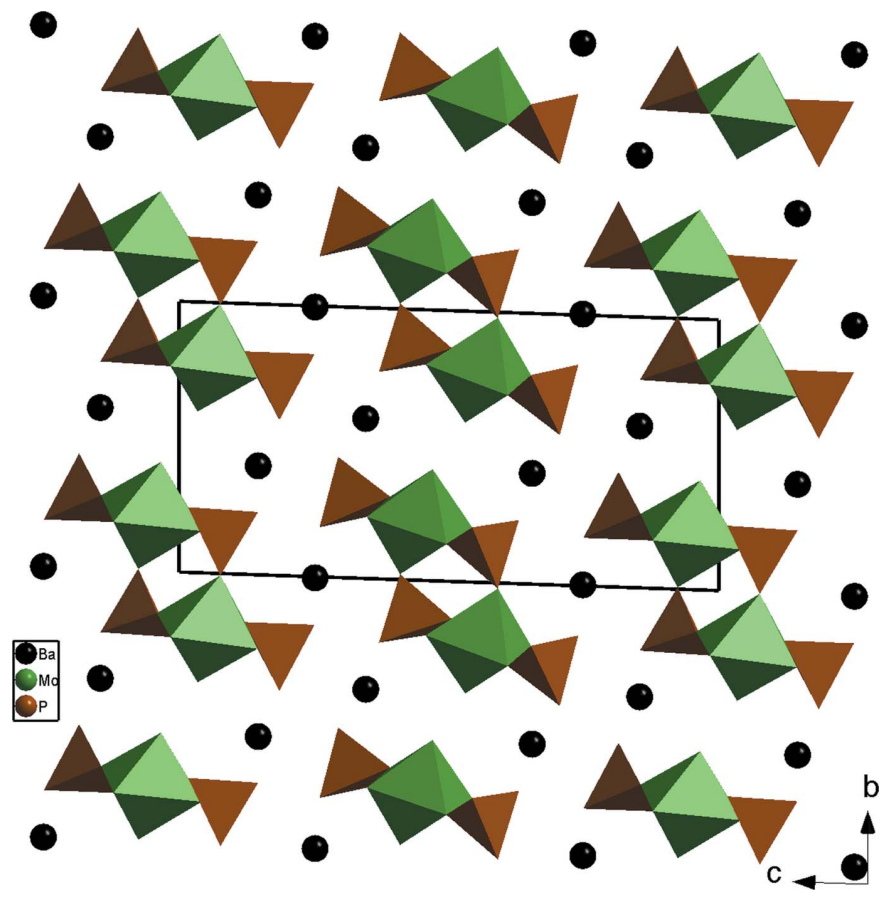

Figure 3

Projection de la structure de $\mathrm{Ba}_{3} \mathrm{MoO}_{2} \mathrm{Mo}_{2}\left(\mathrm{PO}_{4}\right)_{2}$, selon $a$, mettant en évidence l'espace inter-rubans où logent les cations bivalents.

$P 2_{1} / n$ ) les doubles unités cycliques $\mathrm{Mo}_{2} \mathrm{P}_{2} \mathrm{O}_{16}$ se regroupent par partage de sommets entre tétraèdres et formation d'un molybdyldiphosphates, $\mathrm{KMoOP}_{2} \mathrm{O}_{7}$ (Fig. $4 a$ ). Dans les deux variétés non centrosymétriques $\beta$ - $\mathrm{LiMoO}_{2} \mathrm{AsO}_{4}\left(P 2_{1}\right)$ (Hajji et al., 2004) et $\beta-\mathrm{NaMoO}_{2} \mathrm{AsO}_{4}\left(\right.$ Pca2 $\left._{1}\right)$ (Zid et al., 1997), les unités $\mathrm{MoAsO}_{9}$ se connectent pour former des chaînes ondulées de type $\mathrm{MoAsO}_{8}$ qui se lient à leur tour par ponts mixtes de type Mo-O-As selon deux directions pour engendrer des charpentes tridimensionnelles possédant de larges canaux où les cations monovalents résident (Fig. 4b). Le matériau étudié est un nouveau membre de la famille des bismolybdates doubles de formulation $\mathrm{AFe}\left(\mathrm{MoO}_{4}\right)_{2}$ incluant $\mathrm{LiFe}\left(\mathrm{MoO}_{4}\right)_{2}$ (van der Lee et al., 2008), $\mathrm{NaFe}\left(\mathrm{MoO}_{4}\right)_{2}$ (Klevtsova, 1975) et $\mathrm{CsFe}\left(\mathrm{MoO}_{4}\right)_{2}$ (Bazarov et al., 2010). Dans
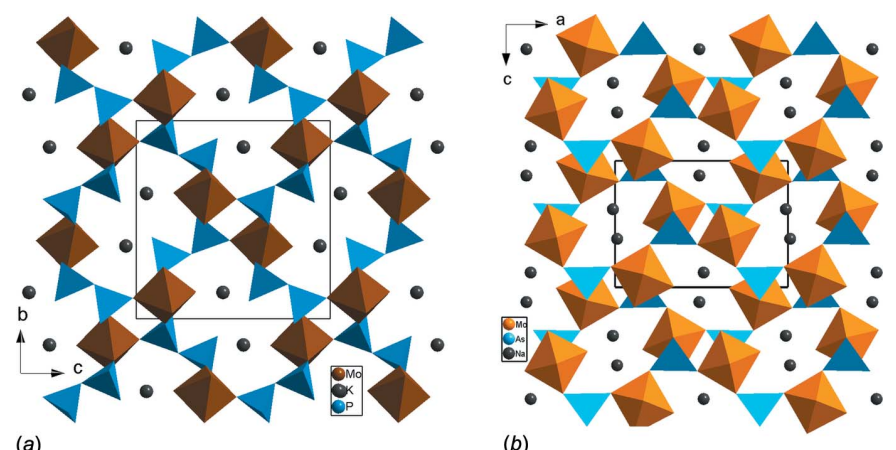

Figure 4

Projections de la structure: $(a)$ de $\mathrm{KMoOP}_{2} \mathrm{O}_{7}$, selon $a$, mettant en évidence la jonction des groupements $\mathrm{Mo}_{2} \mathrm{P}_{2} \mathrm{O}_{16} ;(b)$ de $\beta-\mathrm{NaMoO}_{2} \mathrm{AsO}_{4}$, selon $c$, mettant en évidence la jonction des chaînes ondulées infinie $\mathrm{MoAs}_{2} \mathrm{O}_{8}$.
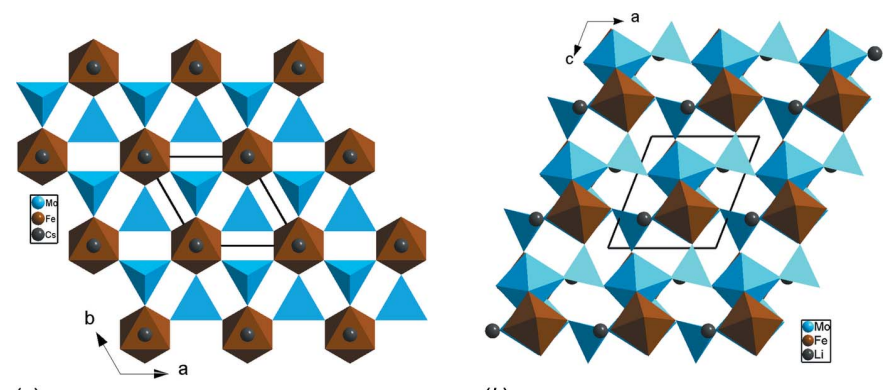

(a)

(b)

Figure 5

Projections de la structure de: $(a) \mathrm{CsFeMo}_{2} \mathrm{O}_{8}$, selon $c$, montrant la jonction des polyèdres par partage de sommets au sein de la couche; $(b)$ $\mathrm{LiFeMo}_{2} \mathrm{O}_{8}$, selon $b$, montrant un autre type de couches dans lesquelles les octèdres partagent des arêtes.

cette famille et pour les structures ayant comme groupe d'espace $P \overline{1}\left[\mathrm{LiFe}\left(\mathrm{MoO}_{4}\right)_{2}\right]$ ou $P m \overline{3} m\left[\mathrm{CsFe}\left(\mathrm{MoO}_{4}\right)_{2}\right]$, les chaînes de type $\mathrm{FeMoO}_{8}$ se lient d'une part par formation de ponts mixtes Fe-O-Mo (Fig. $5 a$ ) et d'autre part par mise en commun d'arêtes entre les octaèdres $M \mathrm{O}_{6}(M=\mathrm{Fe}, \mathrm{Mo})$ et forment un autre type de couches (Fig. $5 b$ ) différent de celui rencontré dans le composé étudié $\mathrm{Ag}_{0.6} \mathrm{Na}_{0.4} \mathrm{Fe}\left(\mathrm{MoO}_{4}\right)_{2}$.

\section{Synthèse et cristallisation}

$\mathrm{Au}$ cours de l'investigation des diagrammes $A-\mathrm{Mo}-\mathrm{Fe}-\mathrm{O}$ $(A=\mathrm{Ag}, \mathrm{Na})$ un nouveau composé de fomulation $\mathrm{Ag}_{0.60} \mathrm{Na}_{0.40} \mathrm{Fe}\left(\mathrm{MoO}_{4}\right)_{2}$ a été élaboré. Les cristaux ont été obtenus à partir des réactifs $\mathrm{AgNO}_{3}$ (Merck, 101510), $\mathrm{Na}_{2} \mathrm{CO}_{3}$ (Fluka, 71350), $\left(\mathrm{NH}_{4}\right)_{2} \mathrm{Mo}_{4} \mathrm{O}_{13}$ (Fluka, 69858) et $\mathrm{FeNO}_{3} \cdot 9 \mathrm{H}_{2} \mathrm{O}$ (Fluka 44949) pris dans les rapports molaires tels que Ag:Na:Fe:Mo restent égaux à 1:1:2:3. Le mélange a été broyé dans un mortier en agate puis placé dans un creuset en porcelaine et préchauffé à $623 \mathrm{~K}$ pour éliminer les produits volatils notamment: $\mathrm{CO}_{2}, \mathrm{NO}_{2}, \mathrm{NH}_{3}$ et $\mathrm{H}_{2} \mathrm{O}$. Le résidu a été finement broyé puis remis dans le four proche de sa fusion à $1173 \mathrm{~K}$. Il est maintenu à cette température pendant trois semaines pour favoriser la germination et la croissance des cristaux. Le magma final est refroidi lentement $(5 \mathrm{~K} / 12 \mathrm{~h})$ jusqu'à $1123 \mathrm{~K}$ puis rapidement $(50 \mathrm{~K} / \mathrm{h})$ jusqu'à la température ambiante. Les cristaux de $\mathrm{Ag}_{0.60} \mathrm{Na}_{0.40} \mathrm{Fe}\left(\mathrm{MoO}_{4}\right)_{2}$ ont été séparés du flux à l'eau chaude. Une analyse qualitative au moyen d'un microscope électronique à balayage environnemental de type FEI Quanta 200, a confirmé la présence des éléments chimiques attendus: $\mathrm{Ag}, \mathrm{Mo}, \mathrm{Fe}, \mathrm{Na}$ et l'oxygène.

\section{Résolution et affinement structural}

Un cristal sélectionné sous microscope polarisant, de bonne qualité, a servi pour la collecte des intensités (tableau 2). L'utilisation des contraintes SUMP, EADP et EXYZ autorisées par le programme $S H E L X$, pour le couple d'ions Ag1/ $\mathrm{Na1}$, conduit à des ellipsoïdes bien définis. De plus, les densités d'électrons maximum et minimum restants dans la Fourier- 
Tableau 2

Détails expérimentaux.

\begin{tabular}{|c|c|}
\hline \multicolumn{2}{|l|}{ Données crystallines } \\
\hline Formule chimique & $\mathrm{Ag}_{0,60} \mathrm{FeMo}_{2} \mathrm{Na}_{0,40} \mathrm{O}_{8}$ \\
\hline$M_{\mathrm{r}}$ & 449,65 \\
\hline Système cristallin, groupe d'espace & Monoclinique, $C 2 / c$ \\
\hline Température (K) & 298 \\
\hline$a, b, c(\AA)$ & $9,8310(9), 5,2740(6), 13,617$ (2) \\
\hline$\beta\left({ }^{\circ}\right)$ & $90,334(8)$ \\
\hline$V\left(\AA^{3}\right)$ & $706,01(15)$ \\
\hline$Z$ & 4 \\
\hline Type de rayonnement & Mo $K \alpha$ \\
\hline$\mu\left(\mathrm{mm}^{-1}\right)$ & 7,17 \\
\hline Taille des cristaux (mm) & $0,32 \times 0,24 \times 0,18$ \\
\hline \multicolumn{2}{|l|}{ Collection de données } \\
\hline Diffractomètre & Enraf-Nonius CAD-4 \\
\hline Correction d'absorption & $\psi$ scan (North et al., 1968) \\
\hline$T_{\min }, T_{\max }$ & $0,166,0,287$ \\
\hline $\begin{array}{l}\text { Nombre de réflexions mesurées, } \\
\text { indépendantes et observées } \\
{[I>2 \sigma(I)]}\end{array}$ & $2875,766,726$ \\
\hline$R_{\text {int }}$ & 0,045 \\
\hline$(\sin \theta / \lambda)_{\max }\left(\AA^{-1}\right)$ & 0,638 \\
\hline \multicolumn{2}{|l|}{ Affinement } \\
\hline$R\left[F^{2}>2 \sigma\left(F^{2}\right)\right], w R\left(F^{2}\right), S$ & $0,025,0,072,1,15$ \\
\hline Nombre de réflexions & 766 \\
\hline Nombre de paramètres & 60 \\
\hline Nombre de restraints & 1 \\
\hline$\Delta \rho_{\max }, \Delta \rho_{\min }\left(\mathrm{e} \AA^{-3}\right)$ & $1,36,-1,48$ \\
\hline
\end{tabular}

Programmes informatiques: CAD-4 EXPRESS (Duisenberg, 1992; Macíček \& Yordanov, 1992), XCAD4 (Harms \& Wocadlo, 1995), SHELXS97 et SHELXL97 (Sheldrick, 2008), DIAMOND (Brandenburg \& Putz, 2001), Win GX (Farrugia, 2012) et publCIF (Westrip, 2010).

différence sont acceptables et sont situées respectivement à $0.87 \AA$ de $\mathrm{O} 2$ et à $0.41 \AA$ de Fe1.

\section{Références}

Baur, W. H. (1974). Acta Cryst. B30, 1195-1215.

Bazarov, B. G., Namsaraeva, T. V., Klevtsova, R. F., Anshits, A. G., Vereshchagina, T. A., Glinskaya, L. A., Fedorov, K. N. \& Bazarova, Zh. G. (2010). Kristallografiya, 55, 634-636.

Boilot, J. P., Collin, G. \& Colomban, Ph. (1987). Mater. Res. Bull. 22, 669-676.

Bouzidi, C., Frigui, W. \& Zid, M. F. (2015). Acta Cryst. E71, 69-72.

Brandenburg, K. \& Putz, H. (2001). DIAMOND. Crystal Impact GbR, Bonn, Allemagne.
Brown, I. D. \& Altermatt, D. (1985). Acta Cryst. B41, 244-247.

Daidouh, A., Veiga, M. L. \& Pico, C. (1997). J. Solid State Chem. 130, 28-34.

Duisenberg, A. J. M. (1992). J. Appl. Cryst. 25, 92-96.

D'Yvoire, F., Pintard-Screpl, E. \& Bretey, E. (1986). Solid State Ionics, 502, 18-19.

D'Yvoire, F., Pintard-Screpl, E., Bretey, E. \& de la Rochère, M. (1983). Solid State Ionics, 851, 9-10.

Ennajeh, I., Georges, S., Ben Smida, Y., Guesmi, A., Zid, M. F. \& Boughazala, H. (2015). RSC Adv. 5, 38918-38925.

Farrugia, L. J. (2012). J. Appl. Cryst. 45, 849-854.

Goodenough, J. B., Hong, H. Y. P. \& Kafalas, J. A. (1976). Mater. Res. Bull. 11, 203-220.

Gueho, C., Borel, M. M., Grandin, A., Leclaire, A. \& Raveau, B. (1992). Z. Anorg. Allg. Chem. 615, 104-108.

Hajji, M., Zid, M. F., Driss, A. \& Jouini, T. (2004). Acta Cryst. C60, i76-i78.

Harms, K. \& Wocadlo, S. (1995). XCAD4. Université de Marburg, Allemagne.

Klevtsova, P. F. (1975). Dokl. Akad. Nauk SSSR, 221, 1322-1325.

Kolitsch, U., Maczka, M. \& Hanuza, J. (2003). Acta Cryst. E59, i10i13.

Ledain, S., Leclaire, A., Borel, M. M., Provost, J. \& Raveau, B. (1996). J. Solid State Chem. 125, 147-152.

Lee, A. van der, Beaurain, M. \& Armand, P. (2008). Acta Cryst. C64, i1-i4.

Linde, S. A., Gorbunova, Yu. E., Lavrov, A. V. \& Tananaev, I. V. (1980). Dokl. Akad. Nauk SSSR, 250, 96-99.

Macíček, J. \& Yordanov, A. (1992). J. Appl. Cryst. 25, 73-80.

Masquelier, C., d'Yvoire, F. \& Collin, G. (1995). J. Solid State Chem. 118, 33-42.

Masquelier, C., Padhi, A. K., Nanjundaswamy, K. S. \& Goodenough, J. B. (1998). J. Solid State Chem. 135, 228-234.

North, A. C. T., Phillips, D. C. \& Mathews, F. S. (1968). Acta Cryst. A24, 351-359.

Ouerfelli, N., Guesmi, A., Molinié, P., Mazza, D., Zid, M. F. \& Driss, A. (2007). J. Solid State Chem. 180, 2942-2949.

Padhi, A. K., Nanjundaswamy, K. S. \& Goodenough, J. B. (1997). J. Electrochem. Soc. 144, 1188-1194.

Sanz, F., Parada, C., Rojo, J. M., Ruiz-Valero, C. \& Saez-Puche, R. (1999). J. Solid State Chem. 145, 604-611.

Shannon, R. D. (1976). Acta Cryst. A32, 751-767.

Sheldrick, G. M. (2008). Acta Cryst. A64, 112-122.

Souilem, A., Zid, M. F. \& Driss, A. (2014). Acta Cryst. E70, i9-i10.

Souilem, A., Zid, M. F. \& Driss, A. (2015). Acta Cryst. E71, 597-601.

Westrip, S. P. (2010). J. Appl. Cryst. 43, 920-925.

Wildner, M. (1992). Z. Kristallogr. 202, 51-70.

Zid, M. F., Driss, A. \& Jouini, T. (1997). J. Solid State Chem. 133, 386 390. 


\section{supporting information}

Acta Cryst. (2016). E72, 737-740 [doi:10.1107/S2056989016006654]

Synthèse, étude et validation structurale d'un triple bis-molybdate en couches, $\mathrm{Ag}_{0.60} \mathrm{Na}_{0.40} \mathrm{Fe}\left(\mathrm{MoO}_{4}\right)_{2}$ lié à yavapaiite

\section{Amira Souilem et Mohamed Faouzi Zid}

Computing details

Data collection: CAD-4 EXPRESS (Duisenberg, 1992; Macíček \& Yordanov, 1992); cell refinement: CAD-4 EXPRESS

(Duisenberg, 1992; Macíček \& Yordanov, 1992); data reduction: XCAD4 (Harms \& Wocadlo, 1995); program(s) used to solve structure: SHELXS97 (Sheldrick, 2008); program(s) used to refine structure: SHELXL97 (Sheldrick, 2008);

molecular graphics: DIAMOND (Brandenburg \& Putz, 2001); software used to prepare material for publication: WinGX (Farrugia, 2012) and publCIF (Westrip, 2010).

Silver sodium iron(III) bis[molybdate(VI)]

Crystal data

$\mathrm{Ag}_{0.60} \mathrm{FeMo}_{2} \mathrm{Na}_{0.40} \mathrm{O}_{8}$

$M_{r}=449.65$

Monoclinic, $C 2 / c$

Hall symbol: $-\mathrm{C} 2 \mathrm{yc}$

$a=9.8310(9) \AA$

$b=5.2740(6) \AA$

$c=13.617(2) \AA$

$\beta=90.334(8)^{\circ}$

$V=706.01(15) \AA^{3}$

$Z=4$

$F(000)=826$

$D_{\mathrm{x}}=4.230 \mathrm{Mg} \mathrm{m}^{-3}$

Mo $K \alpha$ radiation, $\lambda=0.71073 \AA$

Cell parameters from 25 reflections

$\theta=10-15^{\circ}$

$\mu=7.17 \mathrm{~mm}^{-1}$

$T=298 \mathrm{~K}$

Prism, green

$0.32 \times 0.24 \times 0.18 \mathrm{~mm}$

\section{Data collection}

Enraf-Nonius CAD-4 diffractometer

Radiation source: fine-focus sealed tube Graphite monochromator $\omega / 2 \theta$ scans

Absorption correction: $\psi$ scan

(North et al., 1968)

$T_{\min }=0.166, T_{\max }=0.287$

2875 measured reflections

766 independent reflections

726 reflections with $I>2 \sigma(I)$

$R_{\text {int }}=0.045$

$\theta_{\max }=27.0^{\circ}, \theta_{\min }=3.0^{\circ}$

$h=-12 \rightarrow 12$

$k=-6 \rightarrow 6$

$l=-17 \rightarrow 17$

2 standard reflections every $120 \mathrm{~min}$ intensity decay: $1.2 \%$

Refinement

Refinement on $F^{2}$

Least-squares matrix: full

$R\left[F^{2}>2 \sigma\left(F^{2}\right)\right]=0.025$

$w R\left(F^{2}\right)=0.072$

$S=1.15$

766 reflections

60 parameters
1 restraint

Primary atom site location: structure-invariant direct methods

Secondary atom site location: difference Fourier map

$w=1 /\left[\sigma^{2}\left(F_{\mathrm{o}}{ }^{2}\right)+(0.0383 P)^{2}+5.253 P\right]$

where $P=\left(F_{\mathrm{o}}{ }^{2}+2 F_{\mathrm{c}}{ }^{2}\right) / 3$ 
$(\Delta / \sigma)_{\max }<0.001$

$\Delta \rho_{\max }=1.36 \mathrm{e} \AA^{-3}$

$\Delta \rho_{\min }=-1.48$ e $\AA^{-3}$
Extinction correction: SHELXL97 (Sheldrick, 2008), $\mathrm{Fc}^{*}=\mathrm{kFc}\left[1+0.001 \mathrm{xFc}^{2} \lambda^{3} / \sin (2 \theta)\right]^{-1 / 4}$

Extinction coefficient: $0.0042(4)$

\section{Special details}

Geometry. All esds (except the esd in the dihedral angle between two 1.s. planes) are estimated using the full covariance matrix. The cell esds are taken into account individually in the estimation of esds in distances, angles and torsion angles; correlations between esds in cell parameters are only used when they are defined by crystal symmetry. An approximate (isotropic) treatment of cell esds is used for estimating esds involving l.s. planes.

Refinement. Refinement of $\mathrm{F}^{2}$ against ALL reflections. The weighted R-factor $\mathrm{wR}$ and goodness of fit $\mathrm{S}$ are based on $\mathrm{F}^{2}$, conventional R-factors $R$ are based on $F$, with $F$ set to zero for negative $F^{2}$. The threshold expression of $F^{2}>2 \operatorname{sigma}\left(\mathrm{F}^{2}\right)$ is used only for calculating R-factors(gt) etc. and is not relevant to the choice of reflections for refinement. R-factors based on $\mathrm{F}^{2}$ are statistically about twice as large as those based on F, and R- factors based on ALL data will be even larger.

Fractional atomic coordinates and isotropic or equivalent isotropic displacement parameters $\left(\AA^{2}\right)$

\begin{tabular}{llllll}
\hline & $x$ & $y$ & $z$ & $U_{\text {iso }} / U_{\text {eq }}$ & Occ. $(<1)$ \\
\hline Mo1 & $0.67543(3)$ & $0.03643(7)$ & $0.61867(3)$ & $0.0095(2)$ & \\
Ag1 & 0.5000 & $0.57001(16)$ & 0.7500 & $0.0214(3)$ & $0.597(4)$ \\
Na1 & 0.5000 & $0.57001(16)$ & 0.7500 & $0.0214(3)$ & $0.403(5)$ \\
Fe1 & 0.5000 & 0.5000 & 0.5000 & $0.0138(3)$ & \\
O1 & $0.5741(3)$ & $0.3200(6)$ & $0.6162(2)$ & $0.0146(6)$ & \\
O2 & $0.8459(3)$ & $0.1020(7)$ & $0.5812(3)$ & $0.0180(7)$ & \\
O3 & $0.6013(3)$ & $0.8108(6)$ & $0.5404(2)$ & $0.0168(7)$ & \\
O4 & $0.6740(4)$ & $0.9128(7)$ & $0.7348(3)$ & $0.0249(8)$ & \\
\hline
\end{tabular}

Atomic displacement parameters $\left(\AA^{2}\right)$

\begin{tabular}{lllllll}
\hline & $U^{11}$ & $U^{22}$ & $U^{33}$ & $U^{12}$ & $U^{13}$ & $U^{23}$ \\
\hline Mo1 & $0.0100(3)$ & $0.0091(3)$ & $0.0095(3)$ & $0.00035(12)$ & $-0.00039(14)$ & $0.00001(12)$ \\
Ag1 & $0.0298(5)$ & $0.0227(5)$ & $0.0116(4)$ & 0.000 & $0.0044(3)$ & 0.000 \\
Na1 & $0.0298(5)$ & $0.0227(5)$ & $0.0116(4)$ & 0.000 & $0.0044(3)$ & 0.000 \\
Fe1 & $0.0135(5)$ & $0.0141(5)$ & $0.0138(5)$ & $0.0005(3)$ & $-0.0009(3)$ & $-0.0013(3)$ \\
O1 & $0.0173(14)$ & $0.0155(15)$ & $0.0109(14)$ & $0.0039(12)$ & $-0.0028(11)$ & $-0.0011(12)$ \\
O2 & $0.0112(14)$ & $0.0208(17)$ & $0.0220(16)$ & $0.0001(13)$ & $0.0030(12)$ & $-0.0076(14)$ \\
O3 & $0.0155(14)$ & $0.0112(15)$ & $0.0238(16)$ & $-0.0010(13)$ & $-0.0031(12)$ & $-0.0041(13)$ \\
O4 & $0.0342(19)$ & $0.0241(19)$ & $0.0163(17)$ & $-0.0013(16)$ & $-0.0025(14)$ & $0.0070(15)$ \\
\hline
\end{tabular}

Geometric parameters $(\AA, \stackrel{o}{)}$

\begin{tabular}{llll}
\hline $\mathrm{Mo} 1-\mathrm{O} 4^{\mathrm{i}}$ & $1.711(4)$ & $\mathrm{Ag} 1-\mathrm{O} 4^{\mathrm{ii}}$ & $2.499(4)$ \\
$\mathrm{Mo} 1-\mathrm{O} 3^{\mathrm{i}}$ & $1.754(3)$ & $\mathrm{Fe} 1-\mathrm{O} 2^{\mathrm{iii}}$ & $1.957(3)$ \\
$\mathrm{Mo} 1-\mathrm{O} 2$ & $1.788(3)$ & $\mathrm{Fe} 1-\mathrm{O} 2^{\mathrm{iv}}$ & $1.957(3)$ \\
$\mathrm{Mo} 1-\mathrm{O} 1$ & $1.797(3)$ & $\mathrm{Fe} 1-\mathrm{O} 1$ & $1.981(3)$ \\
$\mathrm{Ag} 1-\mathrm{O} 1$ & $2.367(3)$ & $\mathrm{Fe} 1-\mathrm{O} 1^{\mathrm{v}}$ & $1.981(3)$ \\
$\mathrm{Ag} 1-\mathrm{O} 1^{\mathrm{ii}}$ & $2.367(3)$ & $\mathrm{Fe} 1-\mathrm{O} 3$ & $1.994(3)$ \\
$\mathrm{Ag} 1-\mathrm{O} 4$ & $2.499(4)$ & $\mathrm{Fe} 1-\mathrm{O} 3^{\mathrm{v}}$ & $1.994(3)$ \\
& & & \\
$\mathrm{O} 4-\mathrm{Mo1}-\mathrm{O}^{\mathrm{i}}$ & $107.33(18)$ & $\mathrm{O} 1-\mathrm{Fe} 1-\mathrm{O} 1^{\mathrm{v}}$ &
\end{tabular}




\begin{tabular}{|c|c|c|c|}
\hline $\mathrm{O} 4 \mathrm{i}-\mathrm{Mo} 1-\mathrm{O} 2$ & $110.48(18)$ & $\mathrm{O} 2^{\mathrm{iii}-\mathrm{Fe} 1-\mathrm{O} 3}$ & $90.32(14)$ \\
\hline $\mathrm{O} 3^{\mathrm{i}}-\mathrm{Mo} 1-\mathrm{O} 2$ & $110.15(15)$ & $\mathrm{O} 2^{\mathrm{iv}}-\mathrm{Fe} 1-\mathrm{O} 3$ & $89.68(14)$ \\
\hline $\mathrm{O} 4-\mathrm{Mo} 1-\mathrm{O} 1$ & $109.08(16)$ & $\mathrm{O} 1-\mathrm{Fe} 1-\mathrm{O} 3$ & $89.60(13)$ \\
\hline $\mathrm{O} 3^{\mathrm{i}}-\mathrm{Mo1}-\mathrm{O} 1$ & $108.97(14)$ & $\mathrm{O} 1^{\mathrm{v}}-\mathrm{Fe} 1-\mathrm{O} 3$ & $90.40(13)$ \\
\hline $\mathrm{O} 2-\mathrm{Mo1}-\mathrm{O} 1$ & $110.75(16)$ & 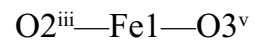 & $89.68(14)$ \\
\hline $\mathrm{O} 1-\mathrm{Mo} 1-\mathrm{Na} 1^{\mathrm{vi}}$ & $116.83(10)$ & $\mathrm{O} 2^{\mathrm{iv}}-\mathrm{Fe} 1-\mathrm{O} 3^{\mathrm{v}}$ & $90.32(14)$ \\
\hline $\mathrm{O} 1-\mathrm{Ag} 1-\mathrm{O}{ }^{\mathrm{ii}}$ & $112.29(16)$ & $\mathrm{O} 1-\mathrm{Fe} 1-\mathrm{O}^{\mathrm{v}}$ & $90.40(13)$ \\
\hline $\mathrm{O} 1-\mathrm{Ag} 1-\mathrm{O} 4$ & $97.21(12)$ & $\mathrm{O} 1^{\mathrm{v}}-\mathrm{Fe} 1-\mathrm{O}^{\mathrm{v}}$ & $89.60(13)$ \\
\hline $\mathrm{O} 1{ }^{\mathrm{ii}}-\mathrm{Ag} 1-\mathrm{O} 4$ & $132.89(11)$ & $\mathrm{O} 3-\mathrm{Fe} 1-\mathrm{O}^{\mathrm{v}}$ & $180.000(1)$ \\
\hline $\mathrm{O} 1-\mathrm{Ag} 1-\mathrm{O} 4^{\mathrm{ii}}$ & $132.89(11)$ & $\mathrm{Mo} 1-\mathrm{O} 1-\mathrm{Fe} 1$ & $127.94(16)$ \\
\hline 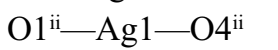 & $97.21(12)$ & $\mathrm{Mo1}-\mathrm{O} 1-\mathrm{Ag} 1$ & $128.48(15)$ \\
\hline $\mathrm{O} 4-\mathrm{Ag} 1-\mathrm{O} 4^{\mathrm{ii}}$ & $87.30(18)$ & $\mathrm{Fe} 1-\mathrm{O} 1-\mathrm{Ag} 1$ & $103.57(13)$ \\
\hline $\mathrm{O} 2^{\mathrm{iii}-}-\mathrm{Fe} 1-\mathrm{O} 2^{\mathrm{iv}}$ & $180.000(1)$ & $\mathrm{Mo} 1-\mathrm{O} 2-\mathrm{Fe}^{\mathrm{vi}}$ & $147.0(2)$ \\
\hline 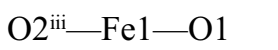 & $87.84(14)$ & $\mathrm{Mo} 1-\mathrm{O} 2-\mathrm{Na} 1^{\mathrm{vi}}$ & $105.22(15)$ \\
\hline $\mathrm{O} 2^{\mathrm{iv}}-\mathrm{Fe} 1-\mathrm{O} 1$ & $92.16(14)$ & 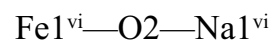 & $91.76(12)$ \\
\hline $\mathrm{O} 2^{\mathrm{iii}}-\mathrm{Fe} 1-\mathrm{O} 1^{\mathrm{v}}$ & $92.16(14)$ & 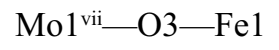 & $158.6(2)$ \\
\hline $\mathrm{O} 2^{\mathrm{iv}}-\mathrm{Fe} 1-\mathrm{O} 1^{\mathrm{v}}$ & $87.84(14)$ & $\mathrm{Mo1}^{\mathrm{vi}}-\mathrm{O} 4-\mathrm{Ag} 1$ & $111.19(18)$ \\
\hline
\end{tabular}

Symmetry codes: (i) $x, y-1, z$; (ii) $-x+1, y,-z+3 / 2$; (iii) $x-1 / 2, y+1 / 2, z$; (iv) $-x+3 / 2,-y+1 / 2,-z+1$; (v) $-x+1,-y+1,-z+1$; (vi) $x+1 / 2, y-1 / 2, z$; (vii) $x, y+1$, $z$. 